\title{
Single excited dual band luminescent hybrid carbon dots-terbium chelate nanothermometer
}

\author{
Rustem R. Zairov ${ }^{a^{*}}$, Alexey P. Dovzhenko ${ }^{b}$, Kirill Sarkanich ${ }^{\mathrm{b}}$, Irek R. Nizameev ${ }^{\mathrm{c}}$, Andrey V. \\ Luzhetskiy $^{\text {d, Svetlana N. Sudakova }}{ }^{\mathrm{a}}$, Sergey N. Podyacheva, Vladimir A. Burilov ${ }^{\mathrm{b}}$, Ivan \\ Vatsouro $^{\mathrm{e}}$, Alberto Vomiero ${ }^{\mathrm{f}, \mathrm{g}}$, Asiya R. Mustafina ${ }^{\mathrm{a}}$
}

\begin{abstract}
${ }^{a}$ Arbuzov Institute of Organic and Physical Chemistry, FRC Kazan Scientific Center, Russian Academy of Sciences, Arbuzov str., 8, 420088, Kazan, Russian Federation.

${ }^{b}$ Kazan (Volga region) Federal University, Kremlyovskaya str., 18, 420008 Kazan, Russian Federation.

${ }^{c}$ Kazan National Research Technical University named after A.N. Tupolev - KAI, 10, K. Marx str., Kazan 420111, Russian Federation.

${ }^{d}$ Federal State Autonomous Educational Institution of Higher Education "Gubkin Russian State University of Oil and Gas" (National Research University)", Leninsky Prospect, 65, 119991, Moscow, Russian Federation.

${ }^{e}$ Department of Chemistry, M. V. Lomonosov Moscow State University, Lenin's Hills

1, 119991 Moscow, Russia.

${ }^{f}$ Department of Molecular Sciences and Nanosystems, Ca' Foscari University Venezia, via Torino 155, 30172 Venezia-Mestre, Italy.

${ }^{g}$ Division of Materials Science, Department of Engineering Sciences and Mathematics, Lulea University of Technology, SE-971 87 Luleå, Sweden.
\end{abstract}

\begin{abstract}
The report introduces hybrid polyelectrolyte-stabilized colloids combining blue and green emitting building blocks, that are citrate carbon dots (CDs) and $[\mathrm{TbL}]^{+}$chelate complexes with 1,3-diketonate derivatives of calix[4]arene. The joint incorporation of green and blue emitting blocks into the polysodium polystyrenesulfonate (PSS) aggregates is carried out through the solvent-exchange synthetic technique. The coordinative binding between $\mathrm{Tb}^{3+}$ centers and $\mathrm{CD}$ surface groups in initial DMF solutions both facilitates joint incorporation of $[\mathrm{TbL}]^{+}$complexes and the CDs into the PSS-based nanobeads and affects fluorescence properties of $[\mathrm{TbL}]^{+}$ complexes and CDs, as well as their ability to temperature sensing. The variation of the synthetic conditions is represented herein as a tool for tuning the fluorescent response of the blue and green emitting blocks upon heating and cooling. The revealed regularities enable developing either dual band luminescent colloids for monitoring temperature changes within $25-50{ }^{\circ} \mathrm{C}$ through double color emission, or to transform the colloids into ratiometric temperature sensors via simple concentration variation of $[\mathrm{TbL}]^{+}$and $\mathrm{CDs}$ in initial DMF solution. Novel hybrid carbon dots-
\end{abstract}


terbium chelate PSS-based nanoplatform opens an avenue for new generation of sensitive and customizable single excited dual band nanothermometers.

Keywords: carbon dots; terbium complex; calix[4]arene; molecular thermometer; nanothermometry; hybrid nanoparticles.

* Author for correspondence. Tel.: +7 843 2734573; fax: +7 8432731872.

E-mail address: rustem02@yandex.ru (R. Zairov). 


\section{Introduction}

Combination of oppositely charged luminophoric blocks with different nature within one nanobead is a promising route to enforce or modify their luminescent response in aqueous solutions. Incorporation of the different luminescent blocks into polymeric nanobead has been widely exemplified by different polymeric matrices ${ }^{1-4}$, including those non-covalently generated from self-aggregation of water soluble polymers ${ }^{5-7}$. Polyelectrolytes provide very promising type of water soluble polymers due to the multitude of surface exposed ionic groups providing high affinity to oppositely charged surfaces or counterions ${ }^{8-10}$. The counterion binding is the wellknown factor facilitating their intra- and inter-molecular interactions, in turn, favoring coil-like conformation of polyelectrolytes followed by their self-assembly into specific clew-like aggregates ${ }^{11}$. However, fundamental backgrounds underlying the use of polyelectrolyte-based aggregates as promising nanobeads for the components with opposite charge and different nature are insufficiently recognized. It is known that the high surface charge of the nanoparticles resulted from the nature of hydrophilic exterior layer and predominantly constituted by chelating anions is the reason for its high affinity to counterions, including metal ions ${ }^{12,13}$. The coordinative binding of CDs with metal ions modifies the fluorescence of CDs, which is the reason for using them as effective optical sensors ${ }^{14-16}$, although an impact of such binding on joint incorporation of blue emitting CDs with green emitting $\mathrm{Tb}^{3+}$ centers into one polyelecrolyte-based nanobead is not enough recognized.

$\mathrm{Ln}^{3+}$-based species possessing narrow emission bands, large Stokes shift, and high emission quantum yields inspired many reports on $\mathrm{Ln}^{3+}$-based thermometers with outstanding sensitivity, temperature resolution, and robustness in the last decade. ${ }^{17,18}$ In this regard, implementation of $\mathrm{Ln}^{3+}$-based materials to hybrid nanostructures is a budding strategy for design of novel ratiometric nanothermometers. Among luminescent nanomaterials, photoluminescent carbon dots have emerged as important materials in sensing and cell imaging. ${ }^{19,20}$ However, most CD-based temperature sensors rely on fluorescence quenching processes, which cannot lead to accurate intracellular thermal sensing due to the influence of many factors such as the presence of metal ions, $\mathrm{pH}$ changes and nonuniform distribution of probes all quenching fluorescence intensity. ${ }^{21}$ All of the drawbacks mentioned above can be mitigated via introducing second not overlapping luminescent component through the built-in calibrations of two emission bands. Thus, for biological samples, which are nonisotropic, it is highly desirable to design novel bicomponent temperature sensors characterized with two well-resolved and intensity comparable luminescence peaks that possess both strong emission and submicron dimensions. 
4

All chemical and biological processes are greatly influenced by temperature, which makes temperature measurements crucial in a big number of practical applications. Conventional temperature sensors, so-called contact thermometers, cannot remotely report the local temperature conditions. Moreover, the explosive growth of interest to micro and nano dimensional objects imposes increasingly stringent requirements in thermometric studies. Real-time response, high spatial resolution in addition to the constraint of having nontoxic and biocompatible devices makes nanothermometry a challenging area of research, in which various functional materials are involved $^{22-24}$. Luminescence-based temperature sensing is outstanding due to its merits of being noninvasive and accurate ${ }^{25-27}$. Therefore, in terms of temperature sensing, luminescence studies of organic dyes ${ }^{28}$, lanthanide-based nanomaterials ${ }^{29,30}$, semiconductor nanocrystals ${ }^{31-34}$, and carbon dots ${ }^{35,36}$ have attracted much attention.

Herein, we report a facile strategy for synthesis of novel hybrid polystyrenesulfonate(PSS)-coated nanoparticles consisting of carbon dots (CDs) and $\mathrm{Tb}^{3+}$ complex as a single excited dual band luminescent nanothermometer. Ligand environment of the $\mathrm{Tb}^{3+}$ complex should fit specific requirements, such as: (1) high complex stability, (2) incomplete coordination sphere of $\mathrm{Tb}^{3+}$ ions, (3) efficient and temperature responsive $\mathrm{Tb}^{3+}$-centered luminescence. The $[\mathrm{TbL}]^{+}$complex with 1,3-diketonate derivatives of calix[4]arene (L) fits well to the aforesaid requirements. The capacity of the hybrid nanoparticles to simultaneously emit in the blue and green regions of spectrum originates from two different emissive components: CDs and $[\mathrm{TbL}]^{+}$complex, correspondingly. The coordinative binding of green emitting $[\mathrm{TbL}]^{+}$complex with blue emitting CDs is highlighted as a tool for their combination within the PSS-nanoplatform as it is schematically demonstrated in Figure 1.

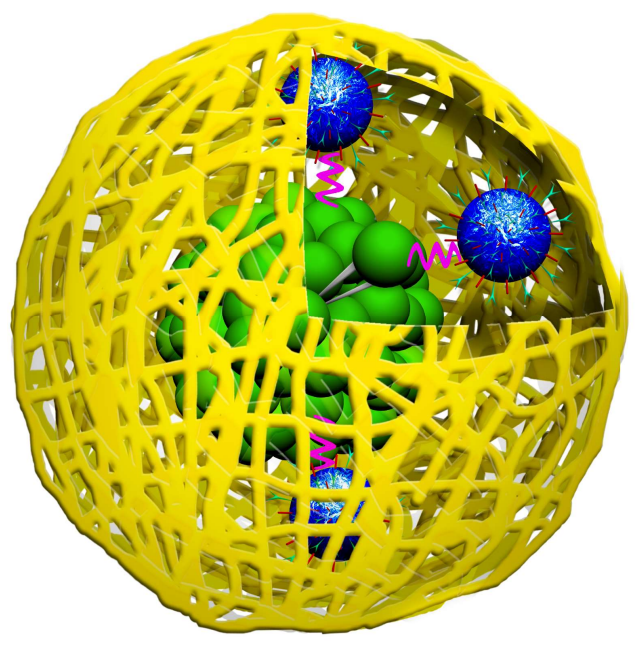

DESCRIPTION

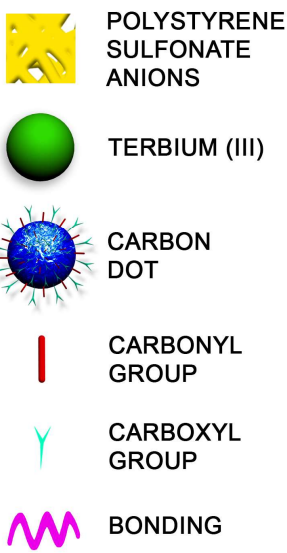

Figure 1. Schematic illustration of PSS- $\{\mathrm{CDs}-[\mathrm{TbL}]\}$ hybrids. 
The variation of the concentration conditions will be introduced as a tool to control mutual influence of the components on their sensing properties in order to develop colloids for temperature monitoring in the $25-50{ }^{\circ} \mathrm{C}$ range through the differently emitting registration channels and to transform the colloids into ratiometric temperature sensors.

\section{Experimental Section}

Materials: citric acid (99\%), ethylenediamine (99\%), $\mathrm{Tb}\left(\mathrm{NO}_{3}\right)_{3} \cdot 6 \mathrm{H}_{2} \mathrm{O}(99.9 \mathrm{wt} \%)$, Triethylamine (TEA) (99\%), N,N-dimethylformamide (DMF), Poly(sodium 4-styrenesulfonate) (PSS) (MWaverage $=70,000)$ and sodium chloride $(\mathrm{NaCl})$ were purchased from Acros Organics. TRIS-buffer, acetic acid, sodium hydroxide, bovine serum albumin (BSA), DMF was twice distilled over $\mathrm{P}_{2} \mathrm{O}_{5}$. Other reagents were used without additional purification.

Tetra-acetylacetonyl derivative of calix[4]arene with hydroxyl substituents (L, 5,11,17,23tetrakis[(acetylaceton-3-yl)methyl)]-25,26,27,28-tetrahydroxy-calix[4]arene, Figure 4a) at its lower rims was synthesized in accordance with the previously reported procedure ${ }^{37}$.

Synthesis of CDs. Carbon quantum dots have been synthesized by microwave synthesis. In a $50 \mathrm{ml}$ flask, $1 \mathrm{~g}$ of citric acid was dissolved in $10 \mathrm{ml}$ of distilled $\mathrm{H}_{2} \mathrm{O}$ and $0.348 \mathrm{ml}$ of ethylenediamine was added. The resulting solution was incubated for 1 minute 30 seconds in a domestic microwave oven until a red caramel residue formed. The product was dissolved in $20 \mathrm{ml}$ of distilled $\mathrm{H}_{2} \mathrm{O}$, filtered from large particles through a membrane $(0.22 \mu \mathrm{m})$ and dried at room temperature.

Synthesis of polyelectrolyte nanoparticles PSS-[TbL]. The $[\mathrm{TbL}]^{+}$complex was obtained via mixing $\mathrm{Tb}\left(\mathrm{NO}_{3}\right)_{3} \cdot 6 \mathrm{H}_{2} \mathrm{O}(\mathrm{C}=0.5 \mathrm{mM})$ with the ligand in a $1: 1$ ratio and adding triethylamine $(\mathrm{C}=3.0 \mathrm{mM})$ to deprotonate the $\beta$-diketonate groups of the calix[4]arene ligand L. PSS-[TbL] nanoparticles were synthesized by adsorption of PSS polyelectrolyte on the surface nanosized templates, obtained by precipitation of the $[\mathrm{TbL}]^{+}$complex from a DMF solution into an aqueous PSS solution $(\mathrm{C}=1 \mathrm{~g} / \mathrm{L}, \mathrm{V}=2.5 \mathrm{ml})$ and $\mathrm{NaCl}(\mathrm{C}=0,5 \mathrm{M})$. To an aqueous solution of PSS was added $0.5 \mathrm{ml}$ of a complex-containing solution of DMF with vigorous stirring $(2200 \mathrm{rpm})$. The resulting colloidal solution was sonicated for 30 minutes while maintaining a constant temperature of $25{ }^{\circ} \mathrm{C}$. Excess PSS was separated by centrifugation at $4{ }^{\circ} \mathrm{C}$ and $15000 \mathrm{rpm}$ for 15 minutes, followed by draining the supernatant.

Synthesis of hybrid nanoparticles PSS- $\{\mathrm{CDs}-[\mathrm{TbL}]\}$. Hybrid PSS- $\{\mathrm{CDs}-[\mathrm{TbL}]\}$ nanoparticles were synthesized on the basis of a DMF solution containing a TbL and CD complex. In the Synthesis_1 concentration of $[\mathrm{TbL}]^{+}-4.5 \mathrm{mM}$ and $\mathrm{CD}-4.5 \mathrm{~g} / \mathrm{L}$ were used. The 
concentrations of the components in the Synthesis_2 were $4.5 \mathrm{mM}$ and $1.5 \mathrm{~g} / \mathrm{L}$ for $[\mathrm{TbL}]^{+}$and CD, respectively. Corresponding DMF solution ([TbL $]^{+}$and $\mathrm{CD}$ mixture) in an amount of $0.5 \mathrm{ml}$ was added to an aqueous solution of $\operatorname{PSS}(\mathrm{C}=1 \mathrm{~g} / \mathrm{L}, \mathrm{V}=2.5 \mathrm{ml})$ and $\mathrm{NaCl}(\mathrm{C}=0,5 \mathrm{M})$ with intensive stirring (2200 rpm). PSS is wound onto the resulting nanoscale $[\mathrm{TbL}]^{+}-\mathrm{CD}$ precipitate with formation a stable colloidal solution. Purification of nanoparticles from excess PSS was carried out by centrifugation under conditions identical to those in the synthesis of PSS-[TbL].

The $\mathrm{pH}$ variations at 4.5-7.0 and 7.0-8.4 were performed by the use of acetic-acetate and TRIS- buffers correspondingly.

The UV-Vis measurements were performed on Lambda 35 spectrophotometer (PerkinElmer, USA) using $10 \mathrm{~mm}$ cuvettes at room temperature. The steady-state emission and excitation spectra were recorded on a fluorescence spectrophotometer Hitachi F-7100 (Japan) with stigmatic concave diffraction grating. Excitation of samples has been performed at $330 \mathrm{~nm}$, and emission detected at $545 \mathrm{~nm}$ with $5 \mathrm{~nm}$ slit width for both excitation and emission. All samples were ultrasonicated for $15 \mathrm{~min}$ before measurements. The dynamic light scattering (DLS) measurements was carried out by means of the Malvern Mastersize 2000 particle analyzer. A He-Ne laser operating at $633 \mathrm{~nm}$ wavelength and emitting vertically polarized light has been used as a light source. The measured autocorrelation functions were analyzed by Malvern DTS software and the second-order cumulant expansion methods. The effective hydrodynamic diameter (d) was calculated by the Einstein-Stokes relation from the first cumulant: $\mathrm{D}=\mathrm{kBT} / 3 \pi \eta \mathrm{d}$, where $\mathrm{D}$ is the diffusion coefficient, $\mathrm{kB}$ is the Boltzmann constant, $\mathrm{T}$ is the absolute temperature, and $\eta$ is the viscosity. The Nano-ZS (MALVERN) with using laser Doppler velocimetry and phase analysis light scattering has been used to determine the zeta potential. The zeta potential was measured at least three times for each sample. Only multiply reproducible results have been taken into account, thereby they differed by less than $4 \%$. The transmission electron microscopy (TEM) images have been obtained with Hitachi HT7700, Japan. The images have been acquired at an accelerating voltage of $100 \mathrm{kV}$. Samples have been sonicated in water for $30 \mathrm{~min}$ and then dispersed on 200 mesh copper grids with continuous formvar support films.

\section{Results and discussion}

The synthesis of hybrid carbon dots-terbium chelate nanothermometer owning blue and green emitting components into the PSS-based nanoplatform was performed through several steps: (1) synthesis of the components; (2) evaluation of their sensing ability to heating and cooling; (3) revealing of the binding between the components; (4) optimization of conditions for the combined incorporation of both components into the PSS-nanoplatform. 
Synthesis, characterization and sensing properties of CDs

Carbon dots (CDs) were synthesized according to a previously reported method in a onestep microwave assisted reaction of citric acid and ethylenediamine. ${ }^{38}$ Transmission electron microscopy (TEM) particle characterization shows that the dots are quasi-spherical monodisperse species with an average size of $(2.3 \pm 0.6) \mathrm{nm}$ remaining erratically distributed on the substrate surface (Figure 2a). The CDs show no tendency to agglomerate upon drying on the formvar coated copper grid used as a substrate.

Dispersion in water at a concentration of $10 \mu \mathrm{g} \mathrm{mL} \mathrm{m}^{-1}$ results in a transparent yellowish aqueous colloids stable for at least three days (Figure S1). UV-Vis absorbance spectroscopy reveals that the water solution of CDs has one distinct absorption bands at $350 \mathrm{~nm}$ and $270 \mathrm{~nm}$ (Figure $2 \mathrm{~b}$ ) assigned to $\mathrm{n} \rightarrow \pi^{*}$ and $\pi \rightarrow \pi^{*}$ transitions of the aromatic $\mathrm{sp}^{2}$ domains, respectively.
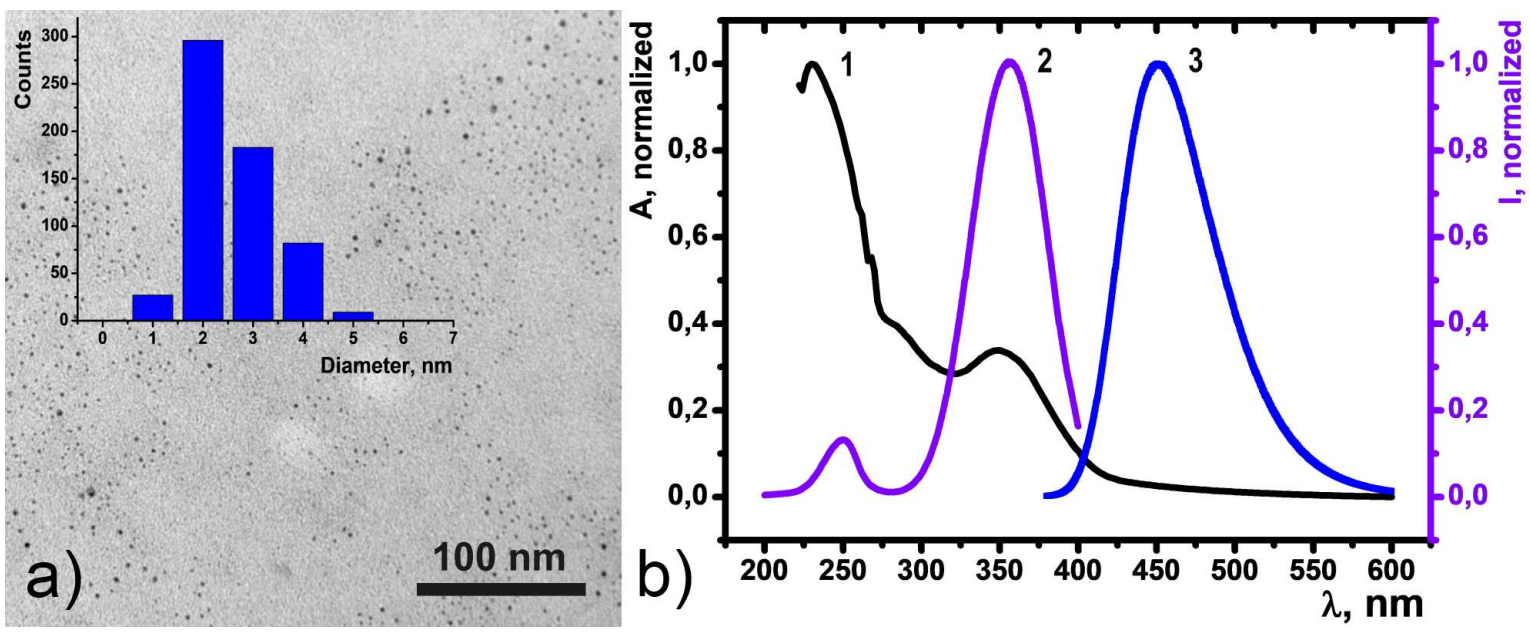

Figure 2. (a) TEM image of dried CDs and corresponding size distribution diagram (inset). (b) UV-Vis absorption spectrum (1), room temperature excitation $\left(2, \lambda_{\mathrm{em}}=450 \mathrm{~nm}\right)$ and fluorescence $\left(3, \lambda_{\mathrm{ex}}=360 \mathrm{~nm}\right)$ spectra of a $10 \mathrm{mg} \mathrm{mL}^{-1} \mathrm{CD}$ aqueous dispersion.

Aqueous colloids of CDs exhibit bright blue fluorescence with the maximum at $450 \mathrm{~nm}$ as a result of $360 \mathrm{~nm}$ xenon lamp excitation (Figure $2 \mathrm{~b}$ ). The color coordinates for luminescence of $\mathrm{CDs}$ in $\mathrm{H}_{2} \mathrm{O}$ are shown on chromaticity diagram (Figure $3 \mathrm{a}$ ). CD emission spectrum was found to be dependent from temperature changes in the range of 25-70 Celsius degrees (Figure 3b). Temperature-fluorescence intensity shows linear response $\left(\mathrm{R}^{2}=0.99577\right.$ for heating; 0.99548 for cooling) over the entire analysis range and the temperature sensitivity was determined to be as high as $1.25 \%{ }^{\circ} \mathrm{C}^{-1}$. This value is comparable with majority of other CD-based nanothermometers ${ }^{35,39-42}$. 
Temperature-dependent fluorescence of the CDs aqueous colloids is fully reversible within $25-70^{\circ} \mathrm{C}$ temperature range (Figure $3 \mathrm{c}$ ). The stability of the CDs colloids under the thermal cycling experiments was evaluated by measuring fluorescence intensity recovery through multiple heating and cooling cycles from $35^{\circ} \mathrm{C}$ to $45^{\circ} \mathrm{C}$ (Figure $3 \mathrm{~d}$ ). The narrowing of the temperature range to $35-45^{\circ} \mathrm{C}$ is aimed at physiological relevant conditions. The thermal reversibility of the CDs was revealed for at least 6 heating-cooling cycles indicating their ability to serve as a fluorescencebased nanothermometer. However, one of the issues of single-parameter thermometers is the need of an external calibration to obtain an absolute value of the temperature through the intensity measurement.
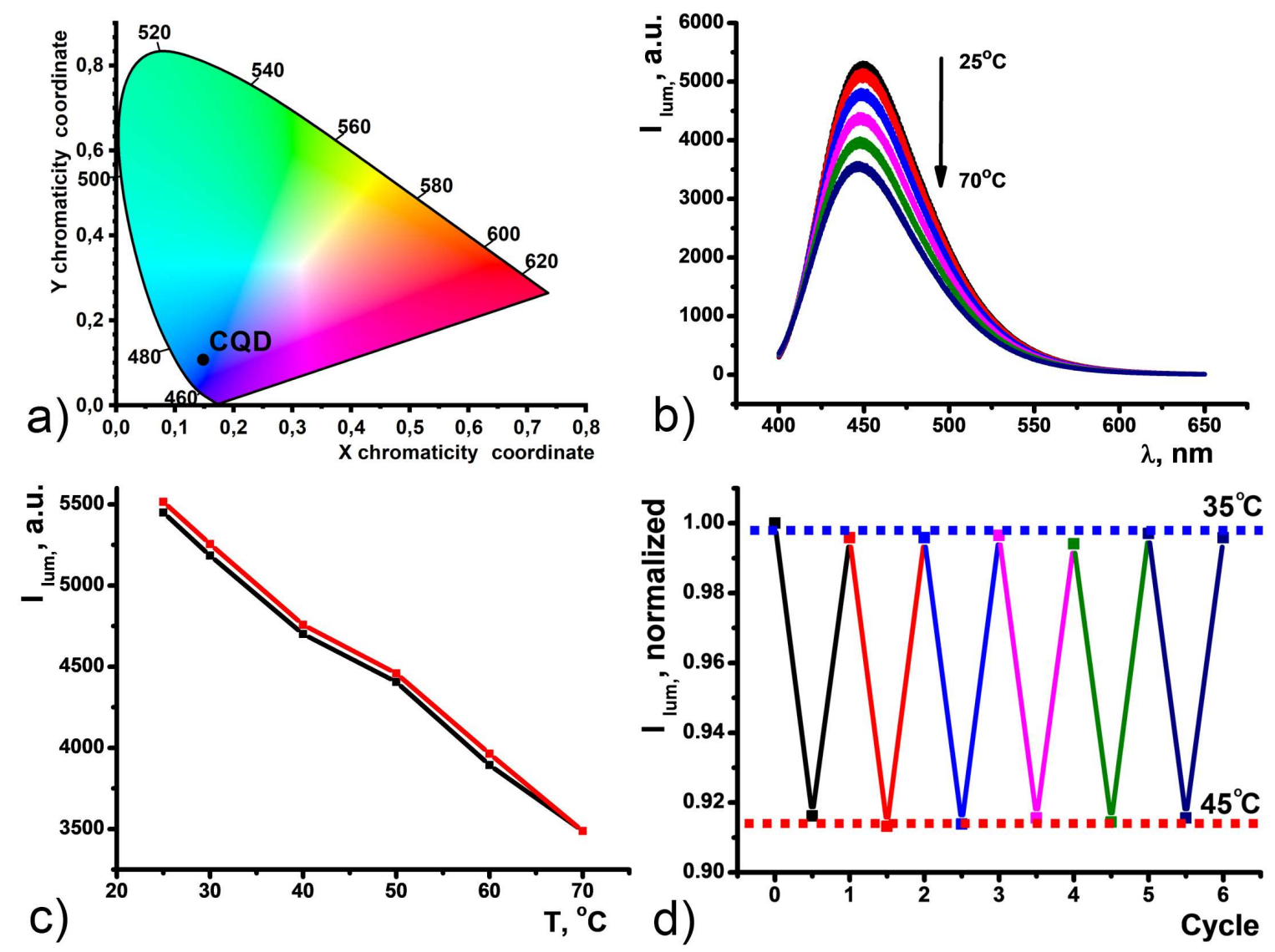

Figure 3. (a) Chromaticity diagram for $\mathrm{CDs}$ in $\mathrm{H}_{2} \mathrm{O}$ (b). Temperature dependence of CDs emission upon heating. (c) Changes in the fluorescence intensity of the CDs $\left(\lambda_{\text {ex }}=360 \mathrm{~nm}\right)$ as a function of temperature over the $25-70^{\circ} \mathrm{C}$ range. (d) Normalized fluorescence intensity upon six heatingcooling cycles of CDs under alternating temperature of $35^{\circ} \mathrm{C}$ and $45^{\circ} \mathrm{C}$.

Synthesis, characterization and sensing properties of PSS-[TbL]

As it was previously reported the synthesis of hydrophilic colloids from the terbium complexes with cyclophanic 1,3-diketonates is based on their efficient self-assembly in the synthetic conditions of the solvent-exchange technique. ${ }^{5-7}$ The $[\mathrm{TbL}]^{+}$complex derives from 
efficient bis-chelating of $\mathrm{Tb}^{3+}$ via two diketonate groups of the tetra-1,3-diketone calix[4]arene (Figures $4 \mathrm{a}, \mathrm{b}$ ). The 1:1 complex is formed in the DMF solutions basified by triethylamine (TEA), which is followed by the sensitization of the $\mathrm{Tb}^{3+}$-centered luminescence due to the efficient ligand-to-metal energy transfer. Both pronounced and reversible temperature-induced changes of the luminescence of the complex in the DMF solutions (Figure S2) are the reason for its choice as the challenging building block of the PSS-stabilized colloids.

The PSS-[TbL] colloids were synthesized through the solvent exchange procedure ${ }^{43}$ (for more details see the Exp. Section). The synthesized colloids were characterized by means of DLS data (Table 1) and TEM images (Figure 4b). The average size revealed from the DLS measurements is about $200 \mathrm{~nm}$ with rather low polydispersity (PDI is below 0.2 ). The negative surface charge manifested by the electrokinetic potential value at $-20 \mathrm{mV}$ confirms that $[\mathrm{TbL}]^{+}$based colloids are embedded into the PSS-aggregates. The difference between the average sizes revealed by TEM and DLS is in agreement with the above-mentioned assumption that PSS-based aggregates incorporate $[\mathrm{TbL}]^{+}$-based colloids.

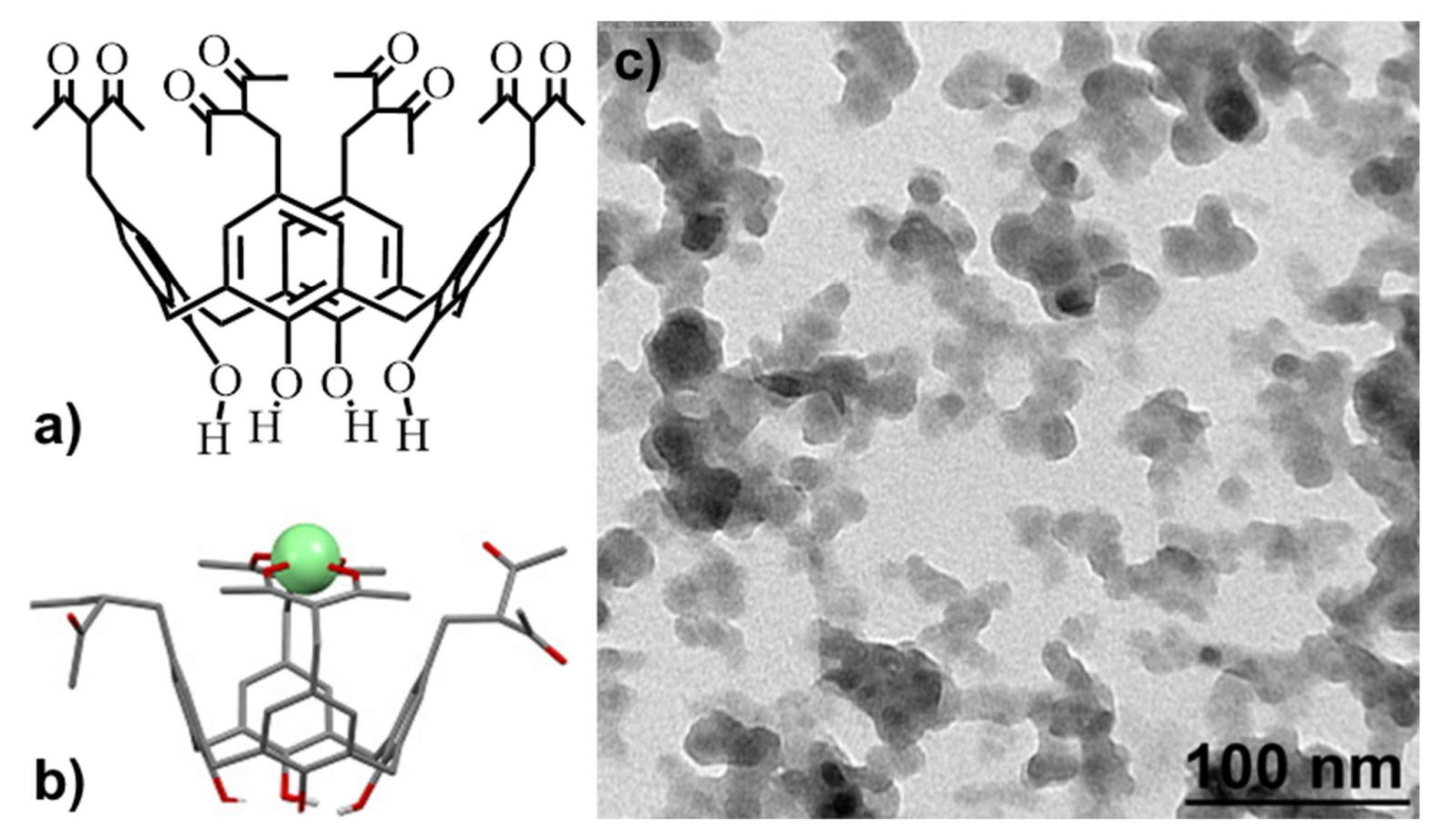

Figure 4. Structure of (a) L and (b) $[\mathrm{TbL}]^{+37}$. (c) TEM image of dried PSS-[TbL] nanoparticles.

The steady state luminescence of PSS-[TbL] nanoparticles is manifested by four narrow bands ${ }^{5} \mathrm{D}_{4} \rightarrow{ }^{7} \mathrm{~F}_{\mathrm{n}}$ peculiar for $\mathrm{Tb}^{3+}$-centered luminescence giving bright green emission with the color coordinates exemplified in Figure 5a. The temperature-dependent luminescence of 
polystyrenesulfonate-coated PSS-[TbL] colloids was studied for the first time here. Gradual decrease of all of four $\mathrm{Tb}$ bands is observed upon heating from $25^{\circ} \mathrm{C}$ to $70^{\circ} \mathrm{C}$ (Figure $5 \mathrm{~b}$ ). The band at $547 \mathrm{~nm}\left({ }^{5} \mathrm{D}_{4} \rightarrow{ }^{7} \mathrm{~F}_{5}\right)$ undergo highest spectral changes ith the sensitivity $\mathrm{S}_{\mathrm{j}}=3.55 \%{ }^{\circ} \mathrm{C}^{-1}$. Cooling the sample back to $25^{\circ} \mathrm{C}$ results in luminescence intensity restoration to initial values. The temperature-dependent luminescence is linear $\left(\mathrm{R}^{2}=0.97593\right.$ for heating; 0.98803 for cooling $)$ and fully reversible as it can be seen from the Figure $5 \mathrm{c}$, where the luminescence under heating or cooling is normalized to the maximum intensity measured at $25^{\circ} \mathrm{C}$. Similarly to CDs, luminescence intensity changes of $547 \mathrm{~nm}$ band were recorded for PSS-[TbL] within $35-45^{\circ} \mathrm{C}$ range. PSS-[TbL] water colloids demonstrate reversibility for at least six cycles (Figure 5d).
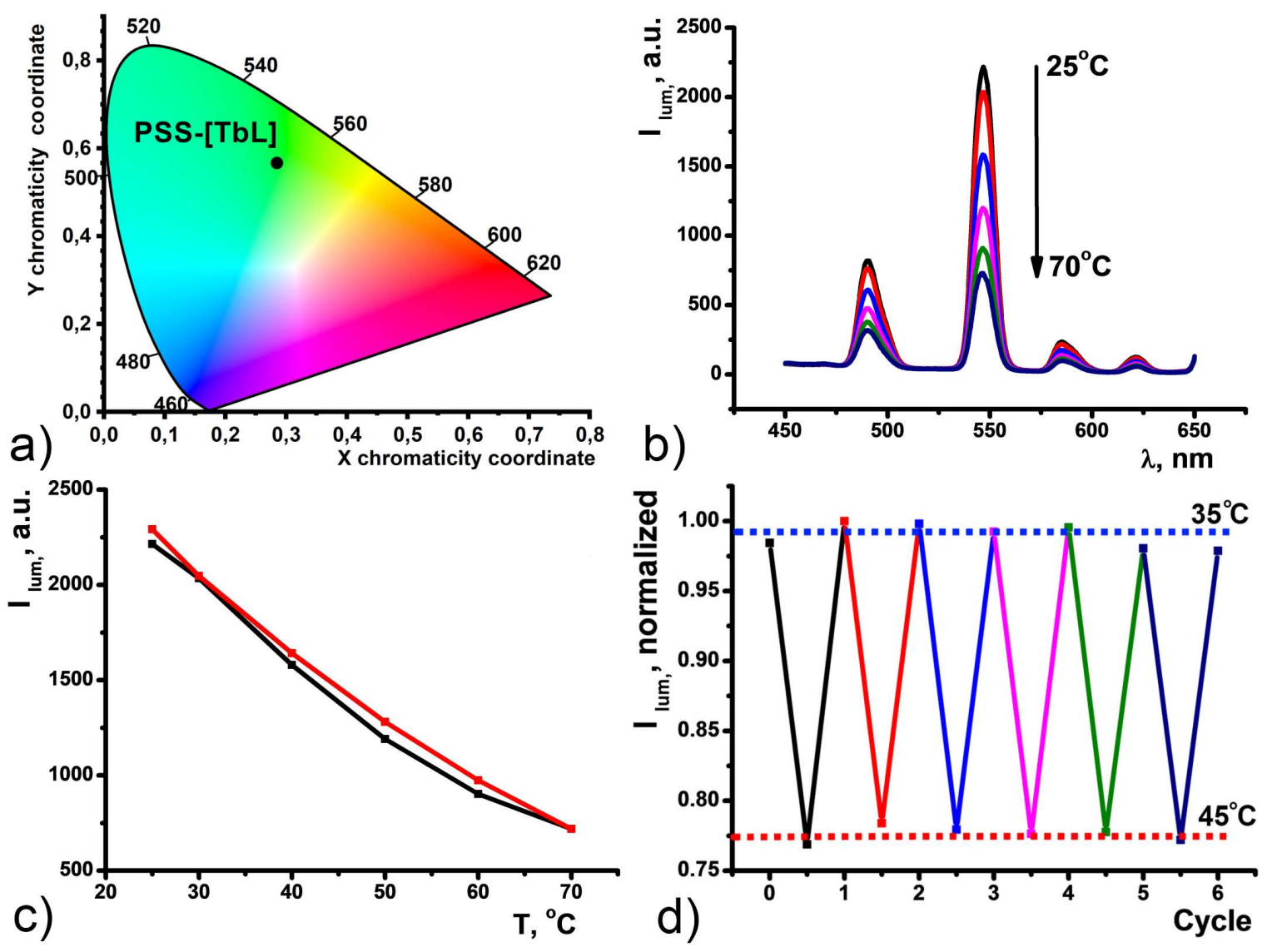

Figure 5. (a) Chromaticity diagram for PSS-[TbL]. (b) Temperature dependence of PSS-[TbL] emission spectrum upon heating. (c) Changes in the fluorescence intensity of the PSS-[TbL] $\left(1_{\mathrm{ex}}\right.$ $=360 \mathrm{~nm}$ ) as a function of temperature over the $25-70^{\circ} \mathrm{C}$ range. (d) Normalized fluorescence intensity upon six heating-cooling cycles of PSS-[TbL] under alternating temperature of $35^{\circ} \mathrm{C}$ and $45^{\circ} \mathrm{C}$.

Relative thermal sensitivities for $[\mathrm{TbL}]^{+}$in DMF, CDs and PSS-[TbL], in aqueous solutions were plotted in Figure 6a. The comparison indicates that the thermal sensitivity of $[\mathrm{TbL}]^{+}$up to $\mathrm{S}_{\mathrm{j}}=7.68 \%{ }^{\circ} \mathrm{C}^{-1}$ at $50^{\circ} \mathrm{C}$ exhibits two-fold lowering to $\mathrm{S}_{\mathrm{j}}=3.55 \%{ }^{\circ} \mathrm{C}^{-1}$ under the transformation of 
$[\mathrm{TbL}]^{+}$to PSS-[TbL] aqueous colloids. Substantial deviation in the thermal sensitivities derives from the difference between the complexes in the DMF solutions and those in the aqueous colloids.
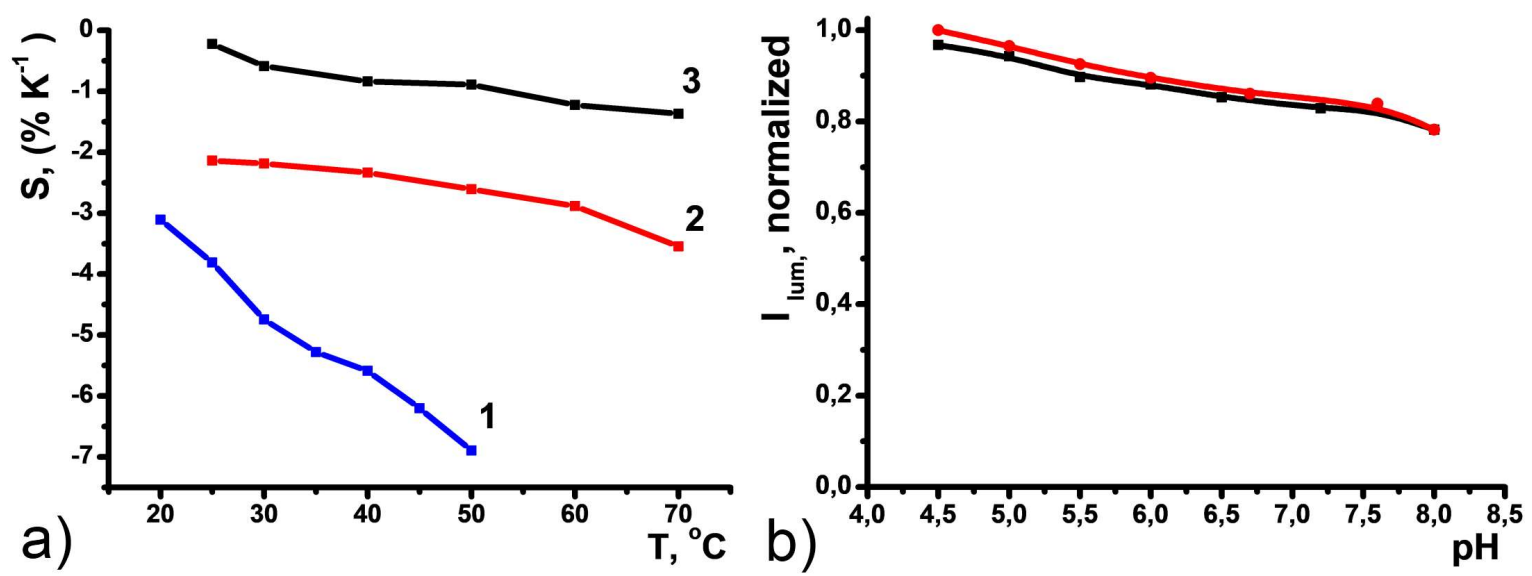

Figure 6. (a) Relative thermal sensitivity for $[\mathrm{TbL}]^{+}$in DMF (1), PSS-[TbL] in $\mathrm{H}_{2} \mathrm{O}$ (2) and CDs in $\mathrm{H}_{2} \mathrm{O}$ (3). (b) Normalized luminescence of PSS-[TbL] versus $\mathrm{pH}$.

Insignificant luminescence intensity dependence of the main $545 \mathrm{~nm}$ band reveals high kinetic stability of PSS-[TbL] in $\mathrm{H}_{2} \mathrm{O}$ to $\mathrm{pH}$ changes in physiological range ( $\mathrm{pH}=4.5-8.0$ ) (Figure $6 b)$. Full reversibility of $\mathrm{I}_{\text {lum }}$ upon basification with $\mathrm{NaOH}$ and acidification with $\mathrm{HCl}$ evidence no $[\mathrm{TbL}]^{+}$degradation, which makes these colloids good basis for the monitoring of temperature changes in biological liquids or tissues known to have different acidity. Luminescence $\lambda_{\mathrm{em}}=450$ $\mathrm{nm}$ band of $\mathrm{CDs}$ was also found to be non-sensitive to $\mathrm{pH}$ changes at $\mathrm{pH}=4.5-8.0$ (Figure $\mathrm{S} 3$ ).

Interactions between $C D$ s and $[T b L]^{+}$in the DMF solutions

It is worth noting that the incorporation of the positively charged complexes into the PSSplatform is electrostatically facilitated, while negative charge of CDs is the factor raising the repulsive interactions with the negatively charges nanobeads. However, the counter-ion binding of the CDs in the basified solutions of $[\mathrm{TbL}]^{+}$can facilitate their joint incorporation into the PSSnanobeads. Thus, interactions between $[\mathrm{TbL}]^{+}$complexes and nanosized carbon dots in the basified DMF solutions should be revealed as the prerequisite of their joint nanoprecipitation in the aqueous-DMF solutions of PSS. The mixing of the components in the basified DMF solutions results in the dual blue-green fluorescence under the excitation by $330 \mathrm{~nm}$ (Figure 7a,b). The spectra represented in Figure $7 \mathrm{~b}$ demonstrates gradual decrease of Tb-centered luminescence intensity upon addition of CDs, which amplifies their interaction with $[\mathrm{TbL}]^{+}$complex. Thus, careful analysis of the fluorescence of each component under concentration variation of another one has been undertaken and demonstrated in Figure 7c,d. 
As it was previously reported, the basification is aimed at shifting the complex formation equilibrium towards $[\mathrm{TbL}]^{+}, 7,37$ where $\mathrm{Tb}^{3+}$ ions are bis-chelated with two 1,3-diketonate moieties. Basified conditions facilitate deprotonation of the surface groups of CDs, which, in turn, is followed by their counter-ion binding with both triethylammonium and $[\mathrm{TbL}]^{+}$cations. The fluorescence of CDs has been studied in the DMF solutions at various concentrations of TEA and $\mathrm{Tb}^{3+}$ ions to recognize and separate their effects. Basification of CDs DMF solution by $80 \mu \mathrm{M}$ of TEA results in $10 \%$ loss of CDs fluorescence intensity (Figure 7c). This fact points to high deprotonation extent of various groups localized on the surface of carbon dots. The addition of $\mathrm{Tb}^{3+}$ ions to TEA rich conditions $(80 \mu \mathrm{M})$ reveals twofold fluorescence intensity quenching of CDs $450 \mathrm{~nm}$ peak, indicating efficient binding with the surface of CDs, which prevents $\mathrm{Tb}^{3+}$ ions from instantaneous hydrolysis (Figure 7c). 

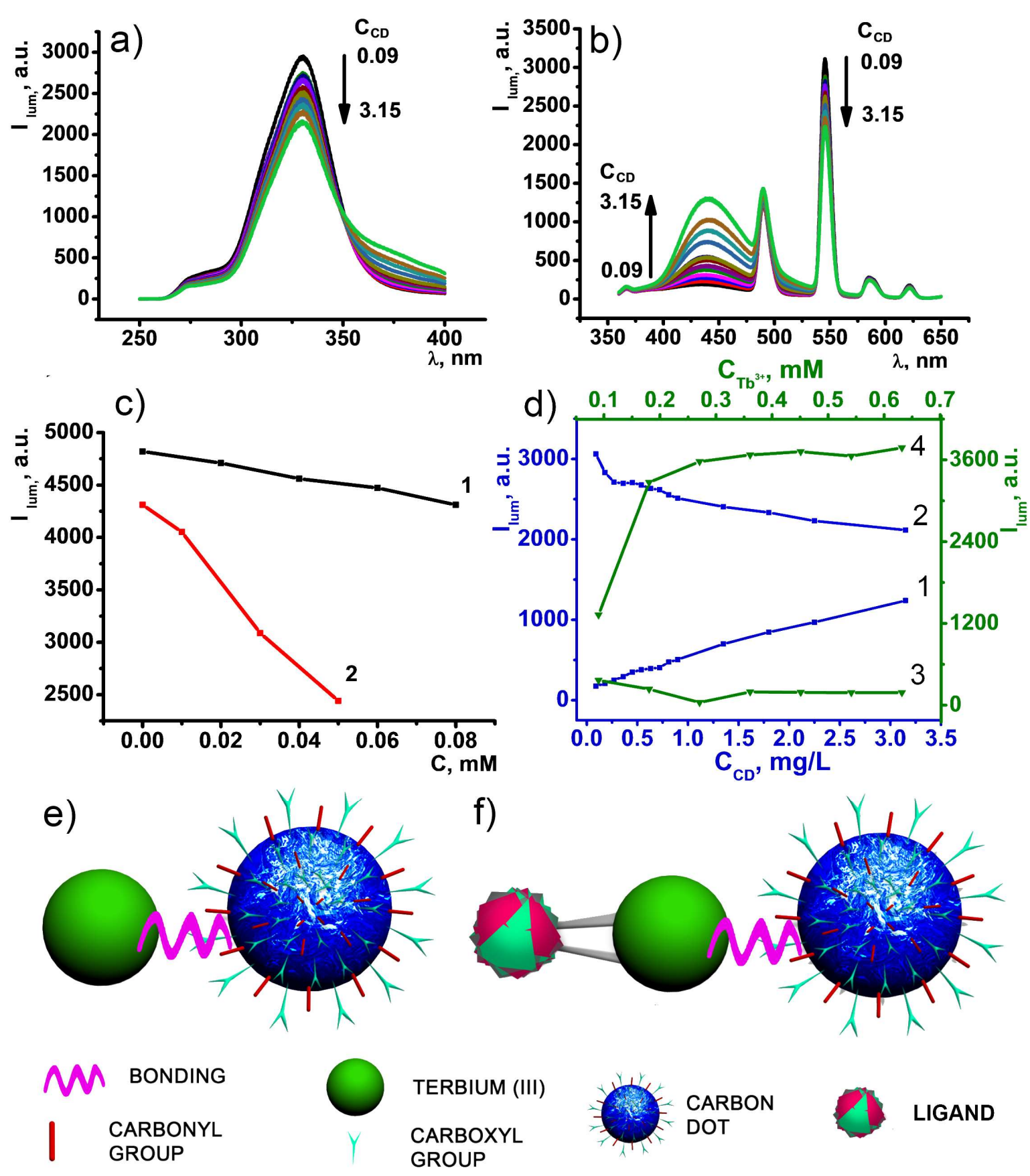

LIGAND

Figure 7. (a) Excitation $\left(\lambda_{\mathrm{em}}=547 \mathrm{~nm}\right)$ and (b) emission $\left(\lambda_{\mathrm{ex}}=330 \mathrm{~nm}\right)$ spectra of CDs and $[\mathrm{TbL}]^{+}$ in $\mathrm{DMF}$ at different $\mathrm{CDs}$ to $[\mathrm{TbL}]^{+}$ratio $\left(\mathrm{C}_{\mathrm{CD}}=0.09-3.15 \mathrm{mg} \cdot \mathrm{L}^{-1} ; \mathrm{C}_{[\mathrm{TbL}]}=0.45 \mathrm{mM}\right)$. (c) Fluorescence intensity of CDs $(0.01 \mathrm{~g} / \mathrm{L})$ versus $\mathrm{C}_{\mathrm{TEA}}(1)$ and versus $\mathrm{C}_{\mathrm{Tb}}(2)$ in the presence of $0.08 \mathrm{mM}$ TEA in DMF. (d) Intensity of $450 \mathrm{~nm}$ (1) and $547 \mathrm{~nm}$ (2) emission peaks as a function of $\mathrm{C}_{\mathrm{CDs}}$ at constant $\mathrm{C}_{[\mathrm{TbL}]}=0.45 \mathrm{mM}$. Intensity of $450 \mathrm{~nm}$ (3) and $547 \mathrm{~nm}$ (4) emission peaks as a function of $\mathrm{C}_{[\mathrm{TbL}]^{+}}$at constant $\mathrm{C}_{\mathrm{CD}}=4.5 \mathrm{mg} \cdot \mathrm{L}^{-1}$ in $\mathrm{DMF}$. (e) Schematic view of Tb-L interaction exemplified by Equillibrium 1. (f) Schematic view of CD-Tb-L interaction exemplified by Equillibrium 2.

The efficient quenching of the $\mathrm{CD}$-centered fluorescence by the $\mathrm{Tb}^{3+}$ ions indicates their efficient complex formation with the chelating anions localized at the surface of CDs. Thus, the 
complex formation should trigger the stripping of the $\mathrm{Tb}^{3+}$ ions from $[\mathrm{TbL}]^{+}$complex which can be introduced schematically by the by Equilibrium 1. However, both complexing ability of the $\mathrm{CDs}$ and incomplete saturation of the inner-coordination sphere of $[\mathrm{TbL}]^{+}$complex allow to assume the probability of the ternary complex formation due to Equilibrium 2. Both Equilibriums are designated in schemes illustrated on Figures 7e,f. The observed changes in the Tb-centered luminescence derive from the interference of equilibriums 1 and 2 , since the luminescence quenching due to equilibrium (1) can be in some extent compensated by the ternary complex formation in accordance with equilibrium (2), since the excited levels of the CDs lie above the excited $\mathrm{Tb}^{3+}$-centered level as it is schematically shown in Figure $\mathrm{S} 4$.

$$
\begin{aligned}
& {[\mathrm{TbL}]+\mathrm{CD} \rightarrow \mathrm{L}+\mathrm{CDTb}} \\
& {[\mathrm{TbL}]+\mathrm{CD} \rightarrow[\mathrm{TbLCD}]}
\end{aligned}
$$

Monitoring of the steady state and time-resolved luminescence of the complex under the varied concentration of the CDs in the DMF solutions can help in choosing optimal concentration to minimize the undesirable destruction of $[\mathrm{TbL}]^{+}$complex and to adjust the intensities of the CDsand $\mathrm{Tb}^{3+}$-centered luminescence on the comparable levels under the same excitation wavelength $(330 \mathrm{~nm})$. The observed losses in the $547 \mathrm{~nm}$ peak intensity of Tb-centered luminescence (Figure $7 \mathrm{~d}$ ) under the increased concentration of the added CDs points to partial degradation of [TbL $]^{+}$ complexes. The excited state lifetime values evaluated from the time-resolved luminescence measurements reveal their insignificant changes under the growing concentrations of CDs at constant $\mathrm{C}_{[\mathrm{TbL}]}=0.45 \mathrm{mM}$ (Table $\mathrm{S} 1$ ). This confirms the partial stripping of $\mathrm{Tb}^{3+}$ ions from $[\mathrm{TbL}]^{+}$ complexes by the CDs as the only reason for the quenching of the luminescence. The quenching of the CD-centered fluorescence under the increased concentration of the added $[\mathrm{TbL}]^{+}$complex also argues for the coordination of either $\mathrm{Tb}^{3+}$ ions or $[\mathrm{TbL}]^{+}$complex with the chelating anions of the CDs. The binding with $\mathrm{Tb}^{3+}$ and $[\mathrm{TbL}]^{+}$as the counterions should induce a surface charge neutralization of the $\mathrm{CDs}$, in turn, facilitating their joint incorporation with $[\mathrm{TbL}]^{+}$complex into the PSS-based aggregates.

\section{Synthesis and characterization of PSS- $\{C D s-[T b L]\}$ colloids}

Synthesis of the hybrid PSS- $\{\mathrm{CDs}-[\mathrm{TbL}]\}$ colloids was performed through the solvent exchange method (see Experimental Section for more details). Variation of the components concentration, $\mathrm{CDs}$ and $[\mathrm{TbL}]^{+}$, in initial DMF solution enables to vary the intensity ratios of 450 $\mathrm{nm}$ and $547 \mathrm{~nm}$ emission peaks upon single excitation by $360 \mathrm{~nm}$ wavelength, correspondingly (Figure 8). Two examples of PSS- $\{\mathrm{CDs}-[\mathrm{TbL}]\}$ hybrids have been synthesized at various $[\mathrm{TbL}]^{+}$ and CDs concentrations. The synthetic conditions $1.5 \mathrm{mM}[\mathrm{TbL}]^{+}$and $1.5 \mathrm{~g} \cdot \mathrm{L}^{-1} \mathrm{CDs}$ for $\mathrm{TbL}$ and 
$\mathrm{CD}$, as well as $4.5 \mathrm{mM}[\mathrm{TbL}]^{+}$and $1.5 \mathrm{~g} \cdot \mathrm{L}^{-1} \mathrm{CDs}$ will be herein and further designated as Synthesis_1 and Synthesis_2. It is worth noting the phase separation through centrifugation as the key step of the synthesis of PSS- $\{\mathrm{CDs}-[\mathrm{TbL}]\}$ colloids, since the colloids of CDs can't be separated from the aqueous solutions. Thus, the appearance of the dual blue-green emitting components in the redispersed after the phase separation aqueous colloids provides clear indication of the joint incorporation of both components into PSS- $\{\mathrm{CDs}-[\mathrm{TbL}]\}$ colloids (Figure 8). The analysis of the dual luminescence of PSS- $\{\mathrm{CDs}-[\mathrm{TbL}]\}$ indicates that the $\mathrm{I}_{547} / \mathrm{I}_{450}$ bands ratio can be varied in a wide range from 1.34 to 8.02 enabling smooth tuning of green and blue contribution. It is worth noting that the concentration variation allows to change the steady state $\mathrm{Tb}^{3+}$ luminescence intensity with remaining the excited state lifetime values unchanged (Table S2), which supports a similarity in the interactions between CDs and $[\mathrm{TbL}]^{+}$within PSS- $\{\mathrm{CDs}-[\mathrm{TbL}]\}$ colloids.
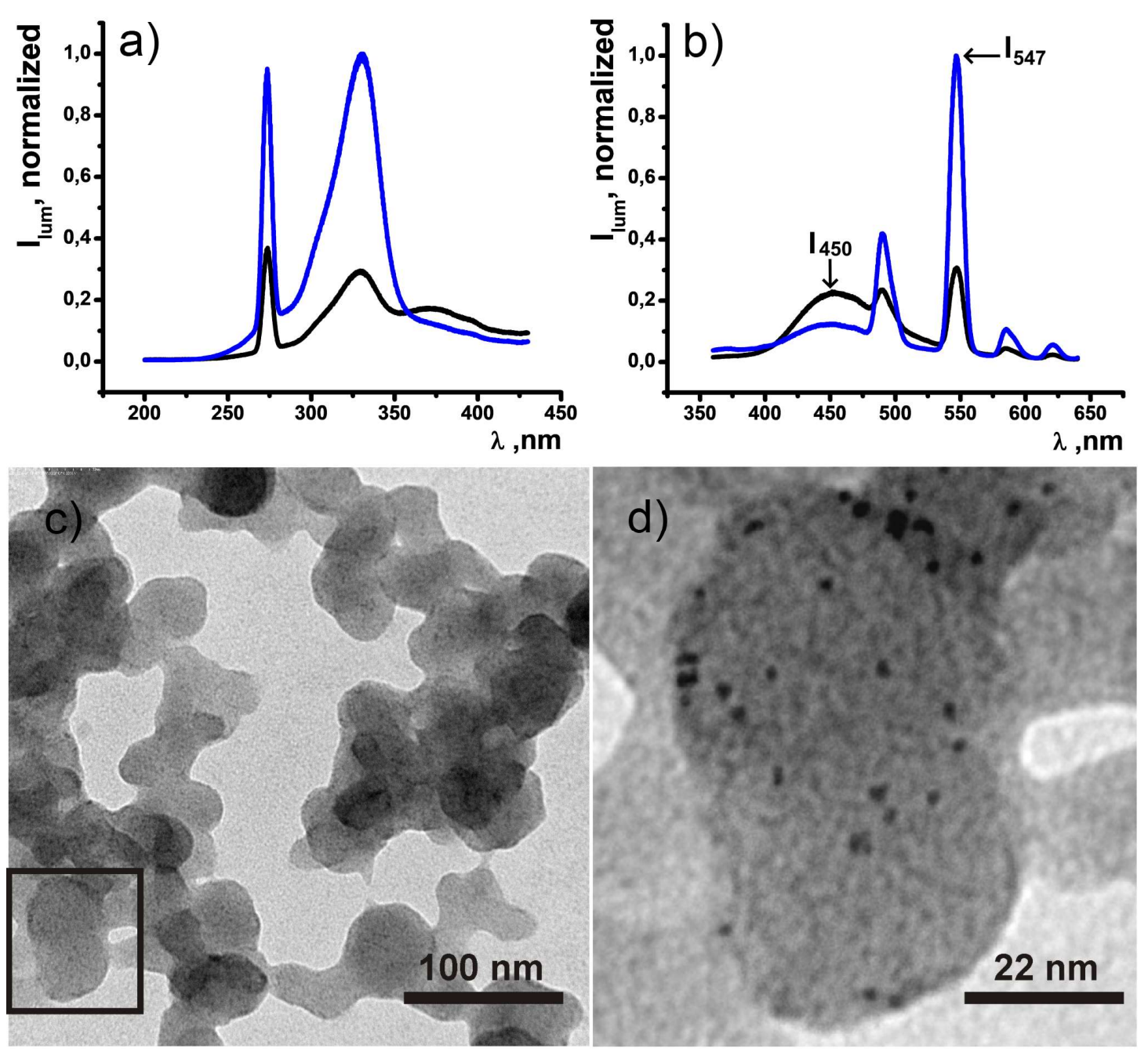

Figure 8. (a) Excitation spectra of PSS- $\{\mathrm{CDs}-[\mathrm{TbL}]\}$ in $\mathrm{H}_{2} \mathrm{O},\left(\lambda_{\mathrm{em}}=450 \mathrm{~nm}\right.$ and $\left.547 \mathrm{~nm}\right)(\mathrm{b})$ Emission spectra of PSS- $\{\mathrm{CDs}-[\mathrm{TbL}]\}$ synthesized according to the Synthesis_1 and Synthesis_2 
$\left(\lambda_{\mathrm{ex}}=360 \mathrm{~nm}\right)$. (c) TEM image of dried PSS- $\{\mathrm{CDs}-[\mathrm{TbL}]\}$ colloids. (d) Enlarged black rectangle area from the Figure 8c.

The TEM image of PSS- $\{$ CDs-[TbL]\} (Synthesis_1) illustrates the presence of worm-like structures formed from globular species intertwined together and layered onto each other upon drying (Figure 8c). The more layers the darker is grey color of worm-like structures formed onto light grey plain color of the formvar grid. Figure $8 \mathrm{~d}$ represents enlarged area of black rectangle from Figure 8c selected for detailed analysis. Black 2-3 nm dots scattered within the structural element of colloidal particles visualize the presence of CDs in PSS- $\{\mathrm{CDs}-[\mathrm{TbL}]\}$ colloids proving hybrid nature of the latter.

Hydrodynamic diameters of PSS- $\{\mathrm{CDs}-[\mathrm{TbL}]\}$ hybrids are higher compared to single PSS[TbL] colloids: $(285.2 \pm 1.2) \mathrm{nm}$ versus $(193.3 \pm 1.2) \mathrm{nm}$; however, PDI values of the hybrid and initial colloids are similar (Table 1). This, along with the higher negative value of zeta potential of the hybrid colloids versus that of the initial colloidal systems (Table 1), provide one more argument for the coexistence of CDs and $[\mathrm{TbL}]^{+}$blocks within each nanoarchitecture.

Table 1. The average hydrodinamic diameters (d), electrokinetic potential values $(\zeta)$ and polydispersity indices (PDI) evaluated from the DLS measurements of PSS- $\{\mathrm{CDs}-[\mathrm{TbL}]\}$ (Synthesis_1).

\begin{tabular}{cccc}
\hline Name & $\mathrm{d}, \mathrm{nm}$ & PDI & Zp, mV \\
\hline PSS[TbL] & $193.3 \pm 1.2$ & $0.171 \pm 0.011$ & $-26.2 \pm 0.6$ \\
PSS- $\{$ CDs-[TbL] $\}$ & $285.2 \pm 1.2$ & $0.258 \pm 0.015$ & $-45.7 \pm 0.7$ \\
CDs & $1648 \pm 523.1$ & $0.951 \pm 0.043$ & $-39.9 \pm 3.7$ \\
\hline
\end{tabular}

\section{Sensing properties of PSS-\{CDs-[TbL]\}}

As it was described in the previous paragraphs, both $\mathrm{CDs}$ and $[\mathrm{TbL}]^{+}$can give explicit luminescent response on temperature variation. Fabrication of novel composite nanomaterial out of two temperature sensitive building blocks embodies the dual band nanothermometer. Different relative sensitivities of blue $(450 \mathrm{~nm})$ and green $(547 \mathrm{~nm})$ emission bands and variable ratio between the building blocks makes it possible color tuning of PSS- $\{\mathrm{CDs}-[\mathrm{TbL}]\}$ depending on the goal of study and complexity of an investigated system. Moreover, the coordinative binding between the CDs and the complexes is the reason for modifying both blue and green luminescent response on temperature changes. In particular, the heating of PSS- $\{\mathrm{CDs}-[\mathrm{TbL}]\}$ (Synthesis_1) up to $70^{\circ} \mathrm{C}$ results in the irreversible quenching of the $\mathrm{Tb}^{3+}$-luminescence, while the temperature responsivity of the CDs components is slightly decreased, but still very strong (Figure 9a). The temperature 
dependence of the dual emission is illustrated by chromaticity diagram in Figure S5. Noteworthy, the green luminescence response of PSS- $\{$ CDs-[TbL] $\}$ (Synthesis_1) is reversible within 34-45 $\mathrm{C}$ and can be recycled for at least 6 times at least (Figure 9b). This means that two registration channels exist for the temperature sensing with the use of PSS- $\{$ CDs-[TbL]\} (Synthesis_1), which can be selected by the researcher in accordance with an object of research.
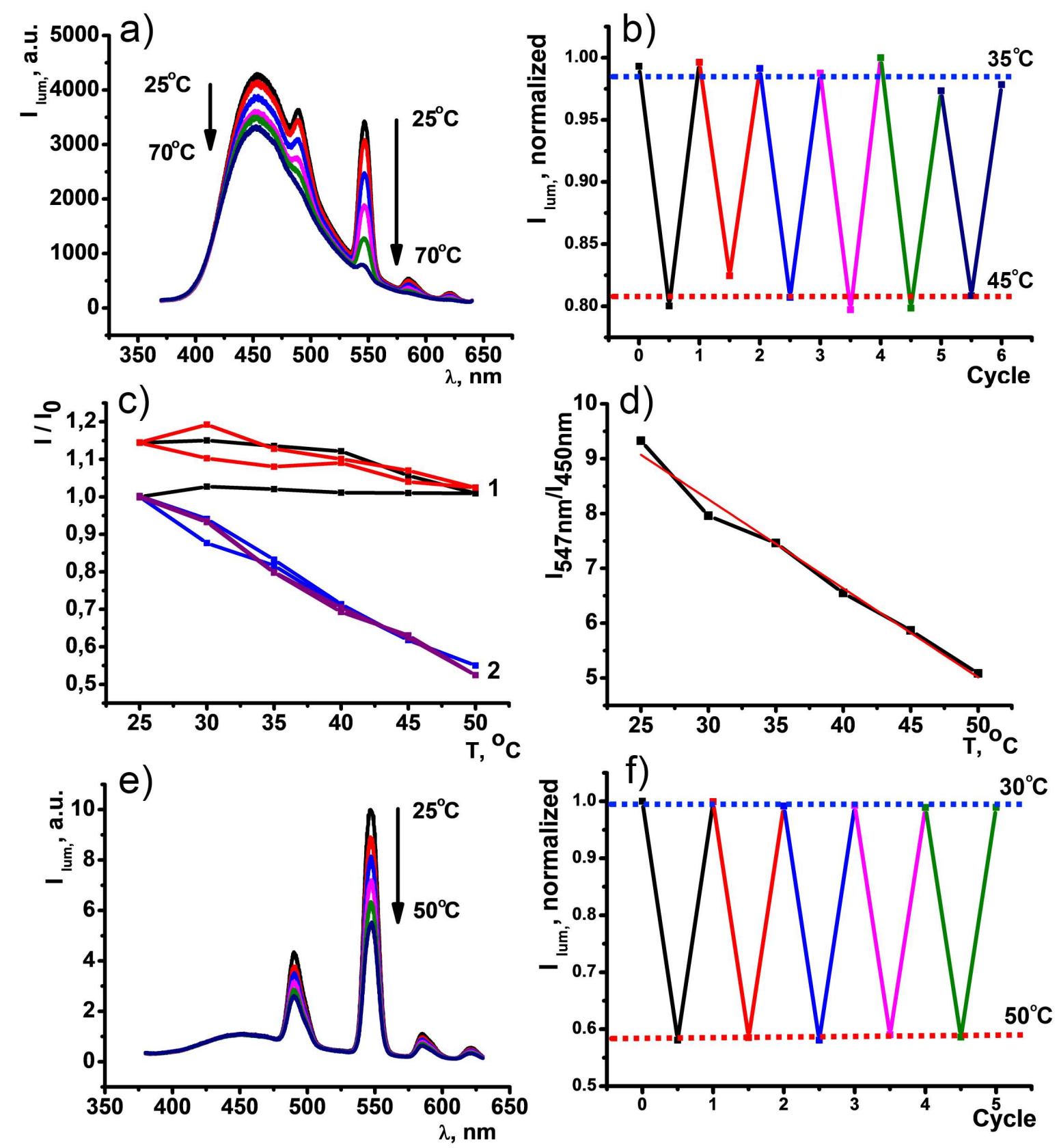

Figure 9. (a) Emission spectra of the PSS- $\{$ CDs-[TbL] $\}$ (Synthesis_1) $\left(1_{\mathrm{ex}}=360 \mathrm{~nm}\right)$ as a function of temperature over the $25-50^{\circ} \mathrm{C}$ range. (b) Temperature dependence of $547 \mathrm{~nm}$ band of PSS$\{\mathrm{CDs}-[\mathrm{TbL}]\}$ (Synthesis_1) upon seven heating-cooling cycles under alternating temperature of $25^{\circ} \mathrm{C}$ and $50^{\circ} \mathrm{C}$. (c) Temperature dependence of $450 \mathrm{~nm}$ (1) and $547 \mathrm{~nm}$ (2) bands of PSS- \{CDs[TbL]\} (Synthesis_2) upon two heating-cooling cycles. (d) $\mathrm{I}_{547} / \mathrm{I}_{450}$ value versus temperature 
within the range of 25-50 Celsius degrees. (e) Emission spectra of the PSS- CDs-[TbL]\} (Synthesis_2) $\left(1_{\mathrm{ex}}=360 \mathrm{~nm}\right)$ as a function of temperature over the $25-50^{\circ} \mathrm{C}$ range. (f) Normalized fluorescence intensity upon four heating-cooling cycles of PSS- $\{\mathrm{CDs}-[\mathrm{TbL}]\}$ (Synthesis_2) under alternating temperature of $25^{\circ} \mathrm{C}$ and $50^{\circ} \mathrm{C}$.

The use of PSS-\{CDs-[TbL]\} (Synthesis_2) enables to transform the colloids to ratiometric nanothermometers for the temperature sensing applications related to inhomogeneous systems, which are majority of biological samples (blood, cells etc.). Minimizing the content of the CDs on going from the colloids (Synthesis_1) to those (Synthesis_2) enables to suppress dramatically the fluorescent response of the CDs to temperature changing (Figure 9e). The binding of the CDs with $\mathrm{Tb}^{3+}$ ions and $[\mathrm{TbL}]^{+}$complexes in greater extent is facilitated by the conditions of Synthesis_2vs Synthesis_1. In turn, the binding event is the reason for disturbance of both fluorescence of the CDs (Figure 7d) and responsivity of the fluorescence on the temperature changes (Figure 9c). The green $547 \mathrm{~nm}$ band in PSS- $\{\mathrm{CDs}-[\mathrm{TbL}]\}$ colloids (Synthesis_2) remains highly sensitive to the changes of temperature in the range of $25-50^{\circ} \mathrm{C}$, while the fluorescence of the CDs exhibit the insignificant changes within the temperature range. This enables to correlate the intensities ratio at 547 and $450 \mathrm{~nm}$ with the temperature values in the range of $25-50^{\circ} \mathrm{C}$, which is demonstrated at Figure 9d. The relative sensitivities $\mathrm{S}_{\mathrm{j}}=2.89 \% \mathrm{C}^{-1}$ are close to each other for the colloids synthesized in both synthetic conditions. Four heating-cooling cycles performed at alternating temperature of $30^{\circ} \mathrm{C}$ and $50^{\circ} \mathrm{C}$ indicate full reversibility of the luminescent response (Figure 9f). It is worth noting the previously reported invariance of the luminescence signal of the PSSstabilized colloids based on the terbium complexes with calix[4]arene 1,3-diketones ${ }^{44}$. This provides a background of invariant luminescence of PSS-\{CDs-[TbL]\} colloids in biological environment, although additional studies, including cell internalization study, intracellular optical thermometry, cell viability tests will be performed in the nearest future and reported elsewhere to outline the applicability of the produced hybrid colloids for the intracellular sensing and/or marking.

\section{Conclusions}

The results introduce joint incorporation of green and blue emitting blocks of different nature exemplified by $[\mathrm{TbL}]^{+}$complexes and CDs into the polysodium polystyrolesulfonate (PSS) aggregates through the solvent-exchange synthetic technique. The coordinative binding between $\mathrm{Tb}^{3+}$ centers and the surface groups of $\mathrm{CDs}$ is the main driving force of their joint incorporation into the PSS-based nanobeads. The binding between the green and blue emitting components affects their fluorescence intensity, as well as the ability to give the fluorescent response to the temperature changes. The revealed regularities both enable to develop the colloids for the 
monitoring of temperature changing within $25-50{ }^{\circ} \mathrm{C}$ through blue and green emitting registration channels and to transform the colloids into ratiometric temperature sensors through the easy variation of the synthetic conditions. Novel hybrid carbon dots-terbium chelate based platform exhibiting single excited dual band emission opens an avenue for new generation of sensitive and customizable nanothermometers.

Author contributions. Rustem R. Zairov, Asiya R. Mustafina Alberto Vomiero Conceptualization, Methodology, Writing - original draft, Writing - review \& editing. Alexey P. Dovzhenko, Kirill Sarkanich, Irek R. Nizameev, Svetlana N. Sudakova, Andrey V. Luzhetskiy, Investigation, Data curation, Formal Analysis. Sergey N. Podyachev, Vladimir A. Burilov, Ivan Vatsouro Resources, Validation, Visualization. Sergey N. Podyachev, Ivan Vatsouro and Rustem R. Zairov Funding acquisition. Asiya R. Mustafina - Supervision.

Conflict of interest statement. There are no conflicts to declare

Acknowledgement. The authors gratefully acknowledge the Assigned Spectral-Analytical Center of FRC Kazan Scientific Center of RAS. Rustem R. Zairov, Sergey N. Podyachev and Ivan Vatsouro thank the Russian Science Foundation (grant 21-13-00115) for financial support. Alberto Vomiero acknowledges the Kempe Foundation and the Knut \& Alice Wallenberg Foundation for financial support.

\section{References.}

1. Jiang, Y. et al. nanoparticles for ultrasensitive in vivo imaging. Nat. Commun. 1-10 doi:10.1038/s41467-019-10119-x.

2. Palner, M., Pu, K., Shao, S. \& Rao, J. Semiconducting Polymer Nanoparticles with Persistent NearInfrared Luminescence for In Vivo Optical Imaging Angewandte. 11639-11642 (2015) doi:10.1002/ange.201502736.

3. Jiang, L. et al. Zuschriften Photodynamic Therapy Luminescent, Oxygen-Supplying, HemoglobinLinked Conjugated Polymer Nanoparticles for Photodynamic Therapy Zuschriften Angewandte. 100049, 10770-10775 (2019).

4. Liu, L. et al. Zero-crosstalk and color-specific photoswitching of dual-emissive polymer nanoparticles for multiple applications. Dye. Pigment. 191, 109370 (2021).

5. Zairov, R. R. et al. Dual red-NIR luminescent Eu Yb heterolanthanide nanoparticles as promising basis for cellular imaging and sensing. Mater. Sci. Eng. C 105, 110057 (2019). 
6. Fedorenko, S. et al. Silica nanoparticles with dual visible-NIR luminescence affected by silica confinement of $\mathrm{Tb}(\mathrm{III})$ and $\mathrm{Yb}(\mathrm{III})$ complexes for cellular imaging application. J. Mater. Sci. 54, 9140-9154 (2019).

7. Podyachev, S. N., Zairov, R. R. \& Mustafina, A. R. 1,3-Diketone Calix[4]arene Derivatives-A New Type of Versatile Ligands for Metal Complexes and Nanoparticles. Molecules 26, 1-29 (2021).

8. Sukhishvili, S. A., Kharlampieva, E. \& Izumrudov, V. Where polyelectrolyte multilayers and polyelectrolyte complexes meet. Macromolecules vol. 39 8873-8881 (2006).

9. Radulescu, A., Murmiliuk, A., Filippov, S. K., Rud, O. \& Košovan, P. Journal of Colloid and Interface Science Reversible multilayered vesicle-like structures with fluid hydrophobic and interpolyelectrolyte layers. 599, 313-325 (2021).

10. Kudryavtseva, V. et al. Micro-sized "pelmeni" - A universal microencapsulation approach overview. Mater. Des. 202, 109527 (2021).

11. Mauser, T., Déjugnat, C. \& Sukhorukov, G. B. Balance of Hydrophobic and Electrostatic Forces in the pH Response of Weak Polyelectrolyte Capsules. J. Phys. Chem. B 110, 20246-20253 (2006).

12. Mahajan, P. G. et al. Chelation enhanced fluorescence of rhodamine based novel organic nanoparticles for selective detection of mercury ions in aqueous medium and intracellular cell imaging. J. Photochem. Photobiol. A Chem. 397, 112579 (2020).

13. Pallares, R. M. et al. Selective Lanthanide Sensing with Gold Nanoparticles and Hydroxypyridinone Chelators. Inorg. Chem. 59, 2030-2036 (2020).

14. Mondal, T. K., Mondal, S., Ghorai, U. K. \& Saha, S. K. White light emitting lanthanide based carbon quantum dots as toxic $\mathrm{Cr}$ (VI) and pH sensor. J. Colloid Interface Sci. 553, 177-185 (2019).

15. Chang, D. et al. Smilax China-derived yellow-fluorescent carbon dots for temperature sensing, Cu2+ detection and cell imaging. Analyst 145, 2176-2183 (2020).

16. Du, F. et al. Facile, rapid synthesis of N,P-dual-doped carbon dots as a label-free multifunctional nanosensor for $\mathrm{Mn}(\mathrm{VII})$ detection, temperature sensing and cellular imaging. Sensors Actuators, $B$ Chem. 277, 492-501 (2018).

17. Cerõn, E. N. et al. Hybrid Nanostructures for High-Sensitivity Luminescence Nanothermometry in the Second Biological Window. Adv. Mater. 27, 4781-4787 (2015).

18. Peng, H. et al. Luminescent europium(III) nanoparticles for sensing and imaging of temperature in the physiological range. Adv. Mater. 22, 716-719 (2010). 
21

19. Cao, L. et al. Carbon Dots for Multiphoton Bioimaging. J. Am. Chem. Soc. 129, 11318-11319 (2007).

20. Baker, S. N. \& Baker, G. A. Luminescent carbon nanodots: Emergent nanolights. Angew. Chemie Int. Ed. 49, 6726-6744 (2010).

21. He, J. et al. Solid-State Carbon Dots with Red Fluorescence and Efficient Construction of DualFluorescence Morphologies. Small 13, 1-10 (2017).

22. Zhou, H., Sharma, M., Berezin, O., Zuckerman, D. \& Berezin, M. Y. Nanothermometry: From Microscopy to Thermal Treatments. ChemPhysChem 17, 27-36 (2016).

23. Bednarkiewicz, A., Marciniak, L., Carlos, L. D. \& Jaque, D. Standardizing luminescence nanothermometry for biomedical applications. Nanoscale 12, 14405-14421 (2020).

24. Carattino, A., Caldarola, M. \& Orrit, M. Gold Nanoparticles as Absolute Nanothermometers. Nano Lett. 18, 874-880 (2018).

25. Brites, C. D. S., Balabhadra, S. \& Carlos, L. D. Lanthanide-based thermometers: at the cuttingedge of luminescence thermometry. Adv. Opt. Mater. 7, 1-30 (2019).

26. del Rosal, B., Ximendes, E., Rocha, U. \& Jaque, D. In Vivo Luminescence Nanothermometry: from Materials to Applications. Adv. Opt. Mater. 5, 1600508 (2017).

27. Ferdinandus et al. Facilely fabricated luminescent nanoparticle thermosensor for real-time microthermography in living animals. ACS Sensors 1, 1222-1227 (2016).

28. De La Rosa, V. R., Woisel, P. \& Hoogenboom, R. Supramolecular control over thermoresponsive polymers. Mater. Today 19, 44-55 (2016).

29. Savchuk, O. A. et al. Upconversion thermometry: A new tool to measure the thermal resistance of nanoparticles. Nanoscale 10, 6602-6610 (2018).

30. Piñol, R. et al. Real-Time Intracellular Temperature Imaging Using Lanthanide-Bearing Polymeric Micelles. Nano Lett. 20, 6466-6472 (2020).

31. Marin, R. et al. Mercaptosilane-Passivated CulnS 2 Quantum Dots for Luminescence Thermometry and Luminescent Labels. ACS Appl. Nano Mater. 2, 2426-2436 (2019).

32. Fanizza, E. et al. Encapsulation of Dual Emitting Giant Quantum Dots in Silica Nanoparticles for Optical Ratiometric Temperature Nanosensors. Appl. Sci. 10, 2767 (2020).

33. Zhao, H., Vomiero, A. \& Rosei, F. Tailoring the Heterostructure of Colloidal Quantum Dots for 
22

Ratiometric Optical Nanothermometry. Small 16, 2000804 (2020).

34. McLaurin, E. J., Vlaskin, V. A. \& Gamelin, D. R. Water-Soluble Dual-Emitting Nanocrystals for Ratiometric Optical Thermometry. J. Am. Chem. Soc. 133, 14978-14980 (2011).

35. Kalytchuk, S. et al. Carbon Dot Nanothermometry: Intracellular Photoluminescence Lifetime Thermal Sensing. ACS Nano 11, 1432-1442 (2017).

36. Sarmanova, O. E. et al. Development of the fluorescent carbon nanosensor for $\mathrm{pH}$ and temperature of liquid media with artificial neural networks. Spectrochim. Acta Part A Mol. Biomol. Spectrosc. 258, 119861 (2021).

37. Zairov, R. et al. Structure impact in antenna effect of novel upper rim substituted tetra-1,3diketone calix[4]arenes on $\mathrm{Tb}(\mathrm{III})$ green and $\mathrm{Yb}$ (III) NIR-luminescence. Tetrahedron 72, 2447-2455 (2016).

38. Du, F., Zeng, F., Ming, Y. \& Wu, S. Carbon dots-based fluorescent probes for sensitive and selective detection of iodide. Microchim. Acta 180, 453-460 (2013).

39. Jaque, D., Martín, E., Martínez, L. \& Haro-, P. Fluorescent nanothermometers for intracellular thermal sensing. Nanomedicine 9, 1047-1062 (2014).

40. Nguyen, V., Yan, L., Xu, H. \& Yue, M. One-step synthesis of multi-emission carbon nanodots for ratiometric temperature sensing. Appl. Surf. Sci. 427, 1118-1123 (2018).

41. Wang, C. et al. Tunable Carbon-Dot-Based Dual-Emission Fluorescent Nanohybrids for Ratiometric Optical Thermometry in Living Cells. ACS Appl. Mater. Interfaces 8, 6621-6628 (2016).

42. Macairan, J. R., Jaunky, D. B., Piekny, A. \& Naccache, R. Intracellular ratiometric temperature sensing using fluorescent carbon dots. Nanoscale Adv. 1, 105-113 (2019).

43. Zairov, R. R. et al. Terbium(III)-thiacalix[4]arene nanosensor for highly sensitive intracellular monitoring of temperature changes within the 303-313 K range. Sci. Rep. 10, 1-13 (2020).

44. Shamsutdinova, N. et al. Tuning magnetic relaxation properties of "hard cores" in core-shell colloids by modification of "soft shell". Colloids Surfaces B Biointerfaces 162, 52-59 (2018). 